\section{O aleitamento materno na pós-graduação em nutrição no Brasil: um perfil das dissertações e teses de 1974 a 2004}

\section{Breastfeeding in post-graduate research on nutrition in Brazil: a profile of dissertations and theses produced between 1974 and 2004}

Raquel Maria Amaral Araújo 1 João Aprígio Guerra de Almeida 2
Departamento de Nutrição. Universidade Federal de Viçosa. Av. PH Holfs, s. n. Campus Universitário. Viçosa, MG, Brasil. CEP: 36.570-000. E-mail: raraujo@ufv.br

2 Instituto Fernandes Figueira. Fundação Oswaldo Cruz. Rio de Janeiro, RJ, Brasil.

\begin{abstract}
Objectives: to trace the profile of academic research on breastfeeding conducted as part of stricto sensu post-graduation courses in nutrition in Brazil.

Methods: a descriptive analysis was carried out of dissertations and theses produced between 1974 and 2004 at all programs recognized by Coordinating Body for the Training of Higher Education Staff.

Results: the programs produced 732 studies in all, of which 48 had breastfeeding as the principal object of study, 42 Masters dissertations and six doctoral theses. The Federal University of Pernambuco had the largest number of publications, while the University of Brasilia and the Federal University of Rio de Janeiro were the ones that, proportionally, addressed the issue most. In 44\% of the programs, no academic research on breastfeeding was found at this level. The programs were especially strong on the specificity of the studies, but tended not investigate cultural aspects, including adoption of the practice of breastfeeding.

Conclusions: nutrition, as an area of scientific knowledge, has not as yet fully embraced the issue of breastfeeding and has failed to address the social and cultural peculiarities the practice of breastfeeding involves.
\end{abstract}

Key words Breastfeeding, Nutrition, Post-graduation

\section{Resumo}

Objetivos: traçar o perfil da produção sobre aleitamento materno na pós-graduação stricto sensu em nutrição no Brasil.

Métodos: análise descritiva das dissertações e teses produzidas no período de 1974 a 2004 por todos os programas reconhecidos pela Coordenação de Aperfeiçoamento de Pessoal de Nivel Superior.

Resultados: os programas produziram 732 obras, das quais 48 utilizaram o aleitamento materno como objeto de construção, sendo 42 em nível de mestrado e seis de doutorado. A Universidade Federal de Pernambuco concentrou o maior número de publicações, enquanto a Universidade de Brasília e a Universidade Federal do Rio de Janeiro foram as que, proporcionalmente, mais se dedicaram a estudar a temática. Em 44\% dos programas não se verificou produção sobre aleitamento materno. Os programas se diferenciaram quanto à especificidade dos estudos, porém não exploraram os aspectos culturais que envolvem a prática do aleitamento materno.

Conclusões: a nutrição, na qualidade de espaço de construção do conhecimento científico, ainda não se encontra, no seu todo, comprometida com a questão do aleitamento materno e não tem se ocupado das peculiaridades sociais e culturais que envolvem sua prática. Palavras-chave Aleitamento materno, Nutrição, Pós-graduação 


\section{Introdução}

As questões que permeiam a alimentação e nutrição de populações têm-se configurado objeto de problematização para a academia, resultando em um elenco variado de teses e dissertações. Nesse cenário se insere a preocupação com a alimentação infantil, sobretudo de lactentes, no qual o aleitamento materno representa unanimidade científica, por se tratar de alimentação segura no início de vida, com repercussões favoráveis na saúde futura, bem como a possibilidade de ganhos psicoafetivos para a mãe e a criança. ${ }^{1-3}$

O número de evidências científicas que qualificam o aleitamento materno como prática inigualável para a alimentação do humano em início de vida segue uma trajetória crescente. Os estudos realizados no campo da alimentação infantil têm gerado o conhecimento para o estabelecimento de diretrizes que norteiam os procedimentos nessa área. Assim é que o aleitamento materno, exclusivo nos seis primeiros meses de vida e continuado até dois anos ou mais, foi oficialmente recomendado pela Organização Mundial de Saúde (OMS) e pelo Ministério da Saúde, por se tratar de uma alimentação cientificamente reconhecida como segura, saudável e sustentável.4,5

A literatura científica brasileira é rica em trabalhos relacionados ao aleitamento materno, desenvolvidos nas diferentes áreas do conhecimento. No campo da saúde, a nutrição é co-responsável no processo de integração de ensino e pesquisa sobre essa temática. Considerando o seu objetivo de proporcionar qualidade de vida, por meio da promoção, manutenção e recuperação da saúde e prevenção de doenças, ${ }^{6}$ presume-se sua participação na produção científica sobre o aleitamento materno. Acresce-se o status que o Brasil ocupa no cenário mundial de promoção dessa prática. Os organismos internacionais de saúde têm reconhecido os esforços realizados pelo país em prol do aleitamento materno, que tem se revelado fator decisivo para a redução da mortalidade infantil. 7

A nutrição, como campo formal de exercício profissional, de ensino e de pesquisa, ou ainda, como política social, constitui um espaço de atividade relativamente recente no mundo, tendo sua emergência no século XX. ${ }^{8}$ No Brasil, a criação dos primeiros cursos de graduação nessa área data do período de 1939 a 1949,8 sendo essa iniciativa resultante de uma aspiração política de desenvolvimento econômico e social do país, extremamente marcada, no âmbito social, pelo assistencialismo.8-10 Inicialmente, esses cursos formavam dietistas que atuavam na assistência ao paciente por meio da alimentação e em programas governamentais de alimentação. ${ }^{9}$ Ressalta-se ainda que a base intelectual desse campo do saber constituiu-se de médicos nutrólogos que definiram conceitos, disciplinas e os conteúdos das ciências da nutrição, com uma forte influência do médico argentino, Pedro Escudero, que difundiu suas concepções acerca da nutrição em toda a América Latina. ${ }^{8-10}$

Ainda que em seu nascedouro a ciência da nutrição tenha constituído a dietética como seu núcleo de identidade, ${ }^{11}$ o campo de atuação do nutricionista ampliou-se no decorrer do tempo, abrangendo hoje as áreas de nutrição clínica, alimentação coletiva, saúde coletiva, nutrição e dietética, ciência e tecnologia de alimentos e educação. 12

A pós-graduação em Nutrição surgiu na década de 70, período de reformulação das ações em prol da nutrição de populações, que resultou no estabelecimento de planos e programas em alimentação e nutrição. Essa nova formatação para as ações de nutrição centralizou-se no Instituto de Alimentação e Nutrição (INAN), responsável por dinamizar o Programa Nacional de Alimentação e Nutrição (PRONAN), cujo objetivo era melhorar a situação nutricional de grupos com risco biológico e social.13 Segundo Lima et al.,14 a implementação da pós-graduação em Nutrição no Brasil se desenvolveu condicionada ao interesse do Estado em integrar pós-graduação e pesquisa ao projeto de nutrição do Brasil. Dessa forma, a pós-graduação na Nutrição, inicialmente, estruturou-se e moldou suas funções formativas e o seu trabalho de investigação e análise conformandose aos propósitos do PRONAN, priorizando temas sobre o grave problema da desnutrição brasileira.

A experiência de pós-graduação da Nutrição tem um percurso de três décadas e, nos últimos anos, o surgimento progressivo de novos programas tem significado um impulso para a expansão de sua atividade científica. 15

Diante do exposto há de se questionar: qual tem sido a contribuição da Nutrição nesse processo de produção do conhecimento científico sobre aleitamento materno? Embora existam estudos sobre a produção científica da Nutrição no Brasil,14,16 não há relato de estudos que investiguem a produção do conhecimento sobre aleitamento materno nesse campo do saber. Na Enfermagem, Oriá et al., ${ }^{17}$ analisaram a produção intelectual dessa área sobre aleitamento materno, com ênfase nas questões teóricas e metodológicas dos estudos publicados em revistas nacionais de enfermagem. Souza 18 pesquisou os determinantes biológicos e sociais influentes na construção do conhecimento sobre a alimentação 
infantil na pediatria brasileira, analisando teses, livros-texto referentes à pediatria e puericultura, e artigos do Jornal de Pediatria.

No sentido de preencher essa lacuna, o presente estudo foi desenvolvido com o objetivo de traçar o perfil da produção sobre aleitamento materno nos programas de pós-graduação stricto sensu em Nutrição no Brasil, reconhecidos pela Coordenação de Aperfeiçoamento de Pessoal de Nível Superior (CAPES), no período de 1974 a 2004.

\section{Métodos}

O estudo foi desenvolvido em três etapas: mapeamento dos programas de pós-graduação stricto sensu em Nutrição reconhecidos no Brasil; identificação e obtenção das dissertações e teses; e análise descritiva do material.

Os programas de pós-graduação stricto sensu em Nutrição e suas respectivas instituições de ensino superior foram localizados no portal da Coordenação de Aperfeiçoamento de Pessoal de Nível Superior (CAPES), na área de Nutrição, integrante da grande área de Ciências da Saúde.

Em seguida, foi solicitada à coordenação de cada programa uma relação completa de toda a produção, que serviu de base para identificar as dissertações e teses sobre aleitamento materno. Por meio do Serviço de Comutação Bibliográfica entre Bibliotecas, foram obtidas cópias dos exemplares sele- cionados.

O estudo corresponde ao período de 1974 a 2004. O ano de 1974 refere-se à data de início da produção do primeiro curso de pós-graduação stricto sensu em Nutrição no Brasil. O ano de 2004 foi estabelecido no sentido de garantir a obtenção de um quantitativo atual e real da produção dos programas. Considerando-se que o espaço de tempo entre a defesa e a entrega da versão final da obra ao respectivo programa pode, em alguns casos, prolongar-se por tempo variável, não foi incluído o ano de 2005.

Para a análise dos temas abordados nas teses e dissertações sobre aleitamento materno utilizou-se, como referência, a classificação de áreas do conhecimento apresentada pelo Conselho Nacional de Desenvolvimento Científico e Tecnológico (CNPq). Nessa classificação, a Nutrição constitui-se em quatro subáreas do conhecimento: Bioquímica da Nutrição; Dietética; Análise Nutricional de População; e Desnutrição e Desenvolvimento Fisiológico. Considerando que a classificação utilizada foi insuficiente quanto à categorização de alguns estudos, foi incluída mais uma subárea proposta pelos autores: Educação nutricional.

\section{Resultados e Discussão}

As instituições de ensino superior envolvidas neste estudo e suas respectivas siglas estão relacionadas na Tabela 1 .

Tabela 1

Instituições de ensino superior com programas de pós-graduação stricto sensu em Nutrição no Brasil reconhecidos pela Coordenação de Aperfeiçoamento de Pessoal de Nível Superior (CAPES), 2004.

\begin{tabular}{|c|c|c|c|}
\hline \multicolumn{4}{|c|}{ Grande Área: Ciências da Saúde } \\
\hline \multicolumn{4}{|c|}{ Área: Nutrição } \\
\hline Programa & Instituição de Ensino Superior & Unidade Federativa & Nível* \\
\hline Nutrição & Universidade Federal de Pernambuco (UFPE) & $\mathrm{PE}$ & $M / D$ \\
\hline Nutrição Humana Aplicada & Universidade de São Paulo (USP) & SP & $M / D$ \\
\hline Nutrição & Universidade Federal de São Paulo (UNIFESP) & SP & $\mathrm{M} / \mathrm{D} / \mathrm{F}$ \\
\hline Ciências da Nutrição & Universidade Federal da Paraíba (UFPB) & PB & $\mathrm{M}$ \\
\hline Nutrição Humana & Universidade de Brasília (UnB) & DF & M \\
\hline
\end{tabular}

* $M=$ Mestrado acadêmico; $D=$ Doutorado; F = Mestrado Profissional.

Fonte: www.capes.gov.br 
Distribuição da produção sobre aleitamento materno dos programas de pós-graduação stricto sensu em Nutrição no Brasil, conforme a origem institucional, 1974 - 2004.

\begin{tabular}{|c|c|c|c|c|c|c|c|c|}
\hline \multirow[t]{3}{*}{ Instituições } & \multicolumn{8}{|c|}{ Produção } \\
\hline & \multicolumn{2}{|c|}{ Dissertações } & \multicolumn{2}{|c|}{ Teses } & \multirow[b]{2}{*}{ Total } & \multicolumn{3}{|c|}{ Aleitamento Materno } \\
\hline & Total & $\begin{array}{l}\text { Produção } \\
\text { sobre } \\
\text { Aleitamento } \\
\text { Materno }\end{array}$ & Total & $\begin{array}{c}\text { Produção } \\
\text { sobre } \\
\text { Aleitamento } \\
\text { Materno }\end{array}$ & & Total & $\begin{array}{c}\% \text { no } \\
\text { Programa }\end{array}$ & $\begin{array}{c}\% \text { do total } \\
\text { dos } \\
\text { Programas }\end{array}$ \\
\hline Universidade Federal de Pernambuco (UFPE) & 260 & 21 & 48 & 2 & 308 & 23 & 7,5 & 48 \\
\hline Universidade Federal do Rio de Janeiro (UFRJ) & 94 & 11 & 0 & 0 & 94 & 11 & 11,7 & 23 \\
\hline Universidade de São Paulo (USP) & 75 & 0 & 19 & 0 & 94 & 0 & 0 & 0 \\
\hline Universidade Federal de São Paulo (UNIFESP) & 96 & 5 & 42 & 4 & 138 & 9 & 6,5 & 19 \\
\hline Universidade Federal da Paraíba (UFPB) & 30 & 2 & 0 & 0 & 30 & 2 & 6,7 & 4 \\
\hline Universidade de Brasília (UnB) & 23 & 3 & 0 & 0 & 23 & 3 & 13,0 & 6 \\
\hline Universidade Federal de Viçosa (UFV) & 14 & 0 & 0 & 0 & 14 & 0 & 0 & 0 \\
\hline Universidade Federal do Rio Grande do Norte (UFRN) & 8 & 0 & 15 & 0 & 23 & 0 & 0 & 0 \\
\hline Universidade Federal de Santa Catarina (UFSC) & 8 & 0 & 0 & 0 & 8 & 0 & 0 & 0 \\
\hline Total & 608 & 42 & 124 & 6 & 732 & 48 & & 100 \\
\hline
\end{tabular}

Até o ano de 2004, período correspondente ao estudo, somavam-se nove programas de pós-graduação stricto sensu em Nutrição, distribuídos geograficamente nas Regiões Sul, Sudeste e Nordeste, conforme a Relação de Cursos Recomendados e Reconhecidos pela CAPES.

No período de 1974 a 2004, os programas de pós-graduação stricto sensu em Nutrição produziram 732 obras, sendo 608 (83\%) dissertações de mestrado e 124 (17\%) teses de doutorado (Tabela 2). $\mathrm{O}$ aleitamento materno foi utilizado como objeto de estudo em $6,6 \%$ do total, o que corresponde a 43 dissertações e cinco teses.

A análise comparativa da produção entre os programas de pós-graduação revelou que a Universidade Federal de Pernambuco (UFPE) detém maior número de publicações sobre aleitamento materno, com $48 \%$ do total produzido sobre essa temática no Brasil no período estudado. Embora esse seja um número expressivo, há de se considerar que a UFPE, primeira instituição a implantar um curso de pós-graduação stricto sensu em Nutrição no Brasil, durante 14 anos foi única responsável pela produção de dissertações da Nutrição, o que lhe confere uma margem mais ampla de possibilidades de produção, comparada à maioria dos programas ainda jovens.

A análise do quantitativo da produção sobre aleitamento materno dentro de cada programa revelou que a Universidade de Brasília (UnB) e a Universidade Federal do Rio de Janeiro (UFRJ) foram as que mais se dedicaram à temática, com cerca de $13,0 \%$ e $12 \%$ de suas respectivas produções.

Os programas de pós-graduação da Universidade de São Paulo (USP), da Universidade Federal de Viçosa (UFV), da Universidade Federal de Santa Catarina (UFSC) e da Universidade Federal do Rio Grande do Norte (UFRN) não dispõem de teses ou dissertações sobre aleitamento materno no período estudado. A produção dos programas depende de suas linhas de pesquisa, que por sua vez são um reflexo do perfil institucional e do tempo necessário para sua consolidação. Neste sentido, vale destacar que a UFV, UFSC e a UFRN implantaram seus programas a partir de 2000. O programa da USP, cuja primeira produção data de 1994, apresenta um longo percurso de produção intelectual sem considerar as questões relacionadas ao aleitamento materno como um problema para a ciência.

$\mathrm{Na}$ Tabela 3 estão relacionados os anos em que ocorreram defesas ou publicações de dissertações e teses sobre aleitamento materno, conforme a instituição de ensino. Todas as instituições apresentaram relativa regularidade na produção sobre a temática ao longo dos anos. Na UFPE o aleitamento materno se configurou como objeto de construção para a pósgraduação desde as primeiras dissertações, assim 
Anos em que ocorreram defesa ou publicação de dissertações e teses sobre aleitamento materno, conforme a instituição de ensino e o ano de criação do programa, 1974 - 2004.

\begin{tabular}{|c|c|c|}
\hline Instituição & $\begin{array}{l}\text { Ano de Criação } \\
\text { do Programa }\end{array}$ & $\begin{array}{l}\text { Ano de Defesa ou de Publicação da } \\
\text { Produção sobre Aleitamento Materno* }\end{array}$ \\
\hline Universidade Federal de Pernambuco (UFPE) & 1971 & $\begin{array}{l}1974 \\
1976(2) \\
1983 \\
1990 \\
1994 \\
1996 \\
1997 \\
1999(3) \\
2000 \\
2001(3) \\
2002(3) \\
2003(2) \\
2004(3)\end{array}$ \\
\hline Universidade Federal do Rio de Janeiro (UFRJ) & 1985 & $\begin{array}{l}1989 \\
1990(3) \\
1991 \\
1999(3) \\
2000 \\
2003(2)\end{array}$ \\
\hline Universidade Federal de São Paulo (UNIFESP) & 1991 & $\begin{array}{l}1995 \\
1996 \\
1997 \\
1999(4) \\
2003(2)\end{array}$ \\
\hline Universidade Federal da Paraíba (UFPB) & 1993 & $\begin{array}{l}2001 \\
2003\end{array}$ \\
\hline Universidade de Brasília (UnB) & 2000 & $2003(3)$ \\
\hline
\end{tabular}

* O número entre parênteses refere-se ao número de defesas ou de publicações naquele ano.

como a UFRJ e UnB. Vale destacar, no caso da UFPE, uma visível interrupção de publicações na segunda metade da década de 80. A Universidade Federal da Paraíba (UFPB), apesar de ter seu programa implantado em 1993, somente em 2001 apresentou a primeira produção sobre aleitamento materno. Um dado interessante é a observação de que a partir de 1989 o volume da produção dos programas é ascendente e, nos quatro primeiros anos de 2000, o total de dissertações e teses é igual à soma do que foi produzido nos dez anos anteriores.

Essa dinâmica observada na cronologia das publicações de dissertações e teses dos programas de nutrição pode guardar relação com o contexto histórico da situação do aleitamento materno no
Brasil na década de 70 e nos anos seguintes a 1980, e com a evolução da pós-graduação em nutrição.

A década de 70, período correspondente à criação da pós-graduação em Nutrição no Brasil, representou o auge do crescimento da indústria de alimentação infantil, que se expandia com um forte apelo para a substituição do leite humano pelo de vaca industrializado. ${ }^{18-20}$ Da mesma forma, a produção científica sobre alimentação infantil crescia e revelava uma marcante presença de estudos envolvendo o uso de leite não humano, o que proporcionava status científico para os substitutos do leite humano. $18 \mathrm{Na}$ pediatria, a conduta médica assumia uma postura favorável ao uso do leite formulado, legitimando-se na literatura científica da época. 18 
Distribuição da produção sobre aleitamento materno dos programas de pós-graduação stricto sensu em Nutrição de instituições de ensino superior brasileiras, segundo as subáreas do conhecimento na nutrição, 1974 - 2004.

\begin{tabular}{|c|c|c|c|c|c|c|}
\hline Instituições & $\begin{array}{l}\text { Bioquímica } \\
\text { da Nutrição* }\end{array}$ & Dietética* & $\begin{array}{l}\text { Análise Nutricional } \\
\text { de População* }\end{array}$ & $\begin{array}{l}\text { Desnutrição e } \\
\text { Desenvolvimento } \\
\text { Fisiológico* }\end{array}$ & $\begin{array}{c}\text { Educação } \\
\text { Nutricional** }\end{array}$ & Total \\
\hline $\begin{array}{l}\text { Universidade Federal de } \\
\text { Pernambuco (UFPE) }\end{array}$ & 5 & 2 & 9 & 4 & 3 & 23 \\
\hline $\begin{array}{l}\text { Universidade Federal do } \\
\text { Rio de Janeiro (UFRJ) }\end{array}$ & 8 & 1 & 2 & 0 & 0 & 11 \\
\hline $\begin{array}{l}\text { Universidade Federal de } \\
\text { São Paulo (UNIFESP) }\end{array}$ & 2 & 0 & 3 & 0 & 4 & 9 \\
\hline $\begin{array}{l}\text { Universidade Federal da } \\
\text { Paraíba (UFPB) }\end{array}$ & 0 & 0 & 2 & 0 & 0 & 2 \\
\hline $\begin{array}{l}\text { Universidade de } \\
\text { Brasília (UnB) }\end{array}$ & 1 & 0 & 0 & 0 & 2 & 3 \\
\hline Total & 16 & 3 & 16 & 4 & 9 & 48 \\
\hline
\end{tabular}

* Classificação segundo critérios do Conselho Nacional de Desenvolvimento Científico e Tecnológico (CNPq); ** Subárea proposta pelos autores para classificação dos temas que não se enquadraram na classificação do CNPq.

Concomitantemente, estudos também mostravam a alta taxa de morbi-mortalidade infantil nas populações pobres dos países da África, Ásia e América Latina,7,18-20 levando a questionamentos sobre a substituição do leite humano na alimentação infantil, tendo em vista suas implicações na saúde da criança. A publicação "O matador de bebês",21 um relatório denunciando a ocorrência da desnutrição infantil ocasionada pela promoção de alimentação artificial no Terceiro Mundo, é um marco no posicionamento frente ao agressivo marketing das multinacionais de leites industrializados.

A década seguinte, de 1980, representou o período de mobilização mundial para a revalorização da amamentação natural, promovida pela OMS e o Fundo das Nações Unidas para a Criança (UNICEF).7,18-20 No Brasil, as repercussões dessa investida foram positivas no sentido da formulação de políticas públicas em favor do aleitamento materno, tendo como base ações de proteção (normas ou leis), promoção (divulgação na comunidade) e apoio (aconselhamento e orientação às mães) a essa prática. ${ }^{7}$ As iniciativas de grande impacto foram a implementação do Programa Nacional de Incentivo ao Aleitamento Materno,20,22 com diversas atividades em prol da amamentação, e aprovação da Norma para Comercialização de Alimentos para Lactentes, que regulamenta a promoção comercial e o uso apropriado dos alimentos colocados à venda como substitutos ou complementos do leite materno, além de mamadeiras, bicos e chupetas.7,23

Esse período foi marcado pela efervescência dos debates e ações de estímulo à prática do aleitamento materno 24 e, conseqüentemente, as instituições de pesquisa foram incitadas a se debruçarem sobre essa questão. Isso poderia explicar a crescente produção científica sobre essa temática na pós-graduação em Nutrição a partir de 1989 , observada no presente estudo.

Outro fato que também pode ter contribuído para esse crescimento, especialmente a partir de 1999, é o surgimento de novos programas, como pode ser observado na Tabela 2, bem como o aumento de pesquisadores capacitados no país entre 1996 e 2003.15,25 Assim, tem-se ampliada a possibilidade de investigações sobre a temática do aleitamento materno, considerando-se o potencial de cientistas 26 e também a crescente demanda por investigações em alimentação infantil. 26

A distribuição das teses e dissertações sobre aleitamento materno, segundo a classificação de subáreas do conhecimento na nutrição, pode ser observada na Tabela 4. A estratificação das publicações por instituições, de acordo com as subáreas mais estudadas, permitiu identificar uma diferenciação dos programas quanto à abordagem dessa temática. Nesse sentido, a UFPE se destaca pelos estudos em análise nutricional de população. A UFRJ, por sua vez, se volta para a bioquímica da 
nutrição, concentrando $73 \%$ de seus estudos nessa subárea. A Universidade Federal de São Paulo (UNIFESP) e a UnB problematizaram a temática privilegiando a educação nutricional, totalizando, respectivamente, $44 \%$ e $67 \%$ de suas publicações. A UFPB dedicou $100 \%$ de suas construções em nível de mestrado à análise nutricional de população.

A UFPE foi a única a apresentar estudos em todas as subáreas. As pesquisas dessa instituição se voltaram prioritariamente para a identificação dos efeitos da desnutrição no período de aleitamento e para a situação epidemiológica do aleitamento materno, especialmente no Nordeste. Dessa forma, as subáreas bioquímica da nutrição, desnutrição e desenvolvimento fisiológico, e análise nutricional de população detiveram o maior número de produções. Essa característica de investigação que se estabeleceu desde as primeiras dissertações e se manteve por todo o período estudado, mostra que essas subáreas emergiram na criação do programa e prevaleceram no decorrer do tempo.

Esse perfil da produção da UFPE, polarizada com as perspectivas biológica e social, revela uma possível influência da inclinação intelectual dos pesquisadores que estabeleceram o campo da nutrição em Pernambuco. Segundo Vasconcelos, 27 duas vertentes intelectuais constituíram o campo da nutrição em Pernambuco, representadas pelos pesquisadores Nelson Chaves e Josué de Castro. Nelson Chaves, reconhecido pelos estudos bioquímicos sobre o valor dos alimentos no Nordeste e análise da desnutrição em Pernambuco, relacionase à vertente biológica, ainda que, posteriormente, tenha ultrapassado esta fronteira para o campo social. A outra vertente é representada por Josué de Castro, considerado iniciador e promotor dos estudos da nutrição na perspectiva social, relacionando a desnutrição com a estrutura social. A predominância de estudos nas subáreas bioquímica da nutrição, desnutrição e desenvolvimento fisiológico e análise nutricional de população no programa da UFPE revela o traço característico desses pesquisadores, definindo o perfil da produção científica naquele ambiente. Conforme Vasconcelos, 28 esses pesquisadores exerceram uma forte influência sobre as gerações de cientistas, especialmente médicos e nutricionistas que, subsequentemente, participaram do processo de institucionalização da nutrição em saúde pública no Brasil.

A UFRJ e a UFPE foram as que mais produziram na subárea bioquímica da nutrição, porém, elas se diferenciaram quanto à abordagem da temática: aleitamento materno. Na UFRJ os estudos se voltam para a composição do leite materno e os aspectos envolvidos na sua produção e utilização. A UFPE, entretanto, buscou identificar os efeitos da desnutrição no período de aleitamento, se preocupando com os aspectos fisiológicos da nutrição.

A nutrição contribuiu amplamente na geração de conhecimento no campo da biologia do aleitamento materno, tanto no que se refere aos aspectos fisiológicos da lactação, como do leite enquanto alimento saudável, seguro e sustentável. Também pesquisou as questões sociais que condicionam o aleitamento materno. $\mathrm{Na}$ área social, o enfoque teóricometodológico que prevalece na nutrição circunscreve-se na epidemiologia, com estudos voltados para o conhecimento da situação do aleitamento materno e seus condicionantes, especialmente no Nordeste. Observa-se, entretanto, ausência de estudos dos aspectos culturais que envolvem a prática de amamentação, especialmente o estudo desse evento a partir da perspectiva da mulher, considerando-se ser essa a protagonista na alimentação da criança.

Ao assumir esta trajetória, os programas reduzem a possibilidade de contribuir para solução de problemas que emergem do social e concorrem para a perpetuação dos elevados índices de desmame precoce, sobretudo no que diz respeito à amamentação exclusiva até o sexto mês de vida do bebê e continuada por dois anos ou mais. A amamentação é um processo alimentar que não pode ser reduzido à dimensão exclusiva do alimento. Para que o leite humano se faça disponível ao lactente, os determinantes biológicos da fisiologia da lactação devem ser facilitados pelos condicionantes sociais que permeiam o cotidiano da mulher que amamenta. Em síntese, a nutrição, preocupada com o leite humano e toda a sua importância como primeiro alimento, não pode perder de vista que a amamentação é uma categoria híbrida 29 que se constrói entre os domínios da biologia e da sociedade, ambos mediados pela aparelhagem psíquica da mulher-mãe que amamenta.

Há de se considerar, entretanto, a possibilidade de produções sobre essa temática realizada por nutricionistas em programas de pós-graduação de outras áreas do conhecimento, em linhas de pesquisa concernentes à nutrição.

\section{Conclusões}

A análise da produção de um programa de pósgraduação stricto sensu permite identificar, entre outras questões, a prioridade conferida às áreas temáticas nas quais se delimitam os objetivos de estudo. As teses e dissertações dos programas de 
pós-graduação stricto sensu em Nutrição refletem, para além da dimensão epistemológica, o grau de articulação das construções acadêmicas com as questões que emergem como problema no cenário da saúde pública. Frente a isso, a ausência de estudos sobre aleitamento materno em $44 \%$ dos programas exige uma reflexão.

As questões de alimentação e nutrição, quando focadas na perspectiva de ecologia do desenvolvimento humano, remetem ao aleitamento materno não apenas como uma prática segura para o lactente, mas como um determinante capaz de reduzir o risco de agravos decorrentes das doenças crônicas não transmissíveis no ciclo da vida. Assim, problematizar a temática do aleitamento materno no intuito de originar objetos de pesquisa que consubstanciem o desenvolvimento de teses e dissertações implica, entre outras, na possibilidade de construir caminhos que viabilizem a adoção de ações preventivas mais eficazes.

Vale ainda destacar que a análise da produção sobre aleitamento materno na pós-graduação em Nutrição revelou uma tendência de se estudar essa temática abordando seus aspectos biológicos e epidemiológicos, sem se preocupar com as questões subjetivas à mulher no processo de amamentação. Essa situação esboça uma visão reducionista da Nutrição acerca da temática. Tal posicionamento pode influenciar a constituição do aleitamento materno como disciplina na academia, e condicionar a atuação profissional, na assistência à amamentação, a uma abordagem restrita ao campo da biologia, em detrimento das especificidades culturais e emocionais da mulher.

\section{Referências}

1. Ferreira EA, Vargas IMA, Rocha SMM. Um estudo bibliográfico sobre o apego mãe e filho: bases para a assistência de enfermagem pediátrica e neonatal. Rev Latino-Am Enfermagem. 1998; 6: 111-6.

2. Giugliani ERJ, Victora CG. Alimentação complementar. J Pediatr (Rio J). 2000; 76 (Supl. 3): 253-62.

3. WHO (World Health Organization). The optimal duration of exclusive breastfeeding. Results of a WHO systematic review. Note for the press 7 [on line] 2001 a April 2: 1-6. Available from: http://www.who.int/inf-pr-2001/en/ note2001-07.html [2005 ago 11]

4. WHO (World Health Organization). Global strategy for infant and young child feeding. In: 54th World Health Assembly; 2001 may 1; Geneva 2001. p. 1-4. (WHA54/Inf.Doc./4).

5. Brasil. Ministério da Saúde. Secretaria de Políticas Públicas de Saúde, Organização Pan Americana da Saúde. Guia alimentar para crianças menores de dois anos. Brasília, DF; 2002.

6. Brasil. Conselho Nacional de Educação, Câmara de Educação Superior. Resolução CNE/CES 5/2001. Diretrizes curriculares nacionais do Curso de Graduação em Nutrição. Diário Oficial da União (DOU) 2001; 9 nov., p. 39, Seção 1.

7. Duas décadas de política pública no Brasil. Gota de leite [on line] 2005. Disponível em: http:www.bvsam.cict. fiocruz.br/gotadeleite/index.htm [2006 abril 20].

8. Vasconcelos FAG. O nutricionista no Brasil: uma análise histórica. Rev Nutr. 2002; 2: 127-38.

9. Bosi MLMA. A face oculta da nutrição: ciência e ideologia. Rio de Janeiro: Editora UFRJ; 1988.

10. Lima ES. Gênese e constituição da educação alimentar: a instauração da norma. Hist Cienc Saúde-Manguinhos. 1998; 5: 57-83.

11. Lima ES. Mal de fome e não de raça: gênese, constituição e ação política da educação alimentar - Brasil - 1934-1946. Rio de Janeiro: Ed. Fiocruz; 2000.
12. Conselho Federal de Nutricionistas. Trinta e um de agosto: dia do nutricionista. Rev Cons Fed. Nutr. 2001; 2: 9-12.

13. Brasil. Conselho de Desenvolvimento Social. Programa Nacional de Alimentação e Nutrição - PRONAN. Brasília, DF; 1976.

14. Lima ES, Oliveira CS, Gomes MCR. Educação nutricional: da ignorância alimentar à representação social na pós-graduação do Rio de Janeiro (1980-98). Hist Cienc SaúdeManguinhos. 2003; 10: 602-35.

15. Kac G, Fialho E, Santos SMC. Panorama atual dos programas de pós-graduação em Nutrição no Brasil. Rev Nutr. 2006; 19: 771-84.

16. Vasconcelos FAG. Os arquivos brasileiros de nutrição: uma revisão sobre produção científica em nutrição no Brasil (1944 a 1968). Cad Saúde Pública. 1999; 15: 303-16.

17. Oriá MOB, Glick DF, Alves MDS. Trends in breastfeeding research by Brazilian nurses. Cad Saúde Pública. 2005; 21 : $20-8$.

18. Souza LMBM. Do leite fraco à biologia da excepcionalidade - as múltiplas faces da mesma moeda [tese doutorado]. Rio de Janeiro: Instituto Fernandes Figueira da Fundação Oswaldo Cruz; 2003.

19. Rea MF. Substitutos do leite materno: passado e presente. Rev Saúde Pública. 1990; 24: 241-9.

20. Brasil. Ministério da Saúde. Instituto Nacional de Alimentação e Nutrição. Programa Nacional de Incentivo ao Aleitamento Materno. Brasília, DF; 1991.

21. Muller M. O matador de bebês. Recife: IMIP; 1995.

22. Rea MF. The Brazilian National Breastfeeding Program: a success story. Int J Gynaecol Obstet. 1990; 31 (Suppl 1): 79-82.

23. Araújo MFM, Rea MF, Pinheiro KA, Schmitz BAS. Avanços na norma brasileira de comercialização de alimentos para idade infantil. Rev Saúde Pública. 2006; 40: 513-20. 
24. Venâncio SI, Monteiro CA. A tendência da prática da amamentação no Brasil nas décadas de 70 e 80 . Rev Bras Epidemiol. 1998; 1: 40-9.

25. Brasil. Ministério da Educação e Cultura. Conselho Nacional de Pós-Graduação. Plano Nacional de PósGraduação - PNPG 2005-2010. Brasília, DF; 2004. [on line]. Disponível em: http://www.propesq.ufpe.br/hp/ fopropne/documentos/Doc_S\%EDntese_19Nov2004.pdf. [2006 abr 12]

26. Brasil. Ministério da Saúde. Secretaria de Atenção à Saúde. Departamento de Atenção Básica. Política nacional de alimentação e nutrição. 2. ed. rev. Brasília, DF; 2003.
27. Vasconcelos FAG. Fome, eugenia e constituição do campo da nutrição em Pernambuco: uma análise de Gilberto Freyre, Josué de Castro e Nelson Chaves. Hist Cienc Saúde-Manguinhos. 2001; 8: 315-39.

28. Vasconcelos FAG. A epidemiologia das deficiências nutricionais no Nordeste: a contribuição de Malaquias Batista Filho à institucionalização da Nutrição em Saúde Pública no Brasil. Cad Saúde Pública. 2000; 16: 533-44.

29. Almeida JAG. Amamentação: um híbrido natureza cultura. Rio de Janeiro: Ed. Fiocruz; 1999.

Recebido em 2 de novembro de 2006

Versão final apresentada em 10 de setembro de 2007

Aprovado em 26 de outubro de 2007 